\title{
Diabetic retinopathy is associated with insulin resistance and cardiovascular autonomic dysfunction in type 2 diabetic patients
}

\author{
Futoshi Anan ${ }^{1}$, Masaki Takayuki ${ }^{2}$, Naohiko Takahashi ${ }^{2}$, Mikiko Nakagawa $^{3}$, Nobuoki Eshima ${ }^{4}$,
} Tetsunori Saikawa ${ }^{3}$ and Hironobu Yoshimatsu ${ }^{2}$

Diabetic retinopathy (DR) and cardiovascular autonomic dysfunction are associated with high mortality in type 2 diabetic patients. This preliminary study was therefore designed to test the hypothesis that DR is associated with insulin resistance and cardiovascular autonomic dysfunction in type 2 diabetic patients without insulin treatment. Seventy persons were diagnosed to have type 2 diabetes in the examination from June 2004 to May 2006. The study group consisted of 29 type 2 diabetic patients with DR (age: $58 \pm 6$ years, mean \pm s.d.) and 41 type 2 diabetic patients with no DR (NDR) ( $n=41,58 \pm 5$ years). Cardiovascular autonomic function was assessed by baroreflex sensitivity (BRS), heart rate variability, plasma norepinephrine concentration and cardiac ${ }^{123}$ I-metaiodobenzylguanidine (MIBG) scintigraphic findings. DR patients had lower BRS, early and delayed ${ }^{123}$ I-MIBG myocardial uptake values and higher percent washout rate (WR) of ${ }^{123}$ I-MIBG than the NDR patients. With respect to metabolic findings, DR patients had higher fasting plasma insulin concentration $(P<0.0001)$ and higher homeostasis model assessment $($ HOMA) index $(P<0.00001)$ than the NDR patients. Multiple logistic regression analysis revealed that the presence of DR was independently predicted by HOMA index and the percent WR of ${ }^{123}$ I-MIBG $(P<0.01$ and $P<0.05$, respectively). Our results suggest that $D R$ is associated with depressed cardiovascular autonomic function and insulin resistance and that HOMA index and the percent WR of ${ }^{123}$ I-MIBG are independently associated with DR in Japanese patients with type 2 diabetes mellitus.

Hypertension Research (2009) 32, 299-305; doi:10.1038/hr.2009.8; published online 27 February 2009

Keywords: baroreflex sensitivity; diabetic retinopathy; ${ }^{123}$ I-metaiodobenzylguanidine scintigraphy; insulin resistance

\section{INTRODUCTION}

There is increasing evidence that micro- and macrovascular complications of diabetes share certain pathophysiological mechanisms. This may explain why microangiopathy has been associated with macroangiopathy and mortality. ${ }^{1,2}$

For example, diabetic retinopathy (DR) has been associated with increased cardiovascular and mortality risk from all causes, particularly in type 2 diabetes. ${ }^{3,4}$

Impaired autonomic nervous activity has been recognized as a crucial component of cardiac dysfunction and is strongly associated with harmful events and overall mortality in diabetic patients. ${ }^{5,6}$ Recently, we have reported that depressed cardiovascular autonomic function is related to insulin resistance in type 2 diabetic patients. ${ }^{7-9}$ Furthermore, DR is reported to be associated with insulin resistance in type 2 diabetic patients. ${ }^{10,11}$ Although these results strongly suggest that $\mathrm{DR}$, insulin resistance and autonomic dysfunction are related to each other, the significance of DR for diabetic cardiovascular autonomic function has not been adequately investigated.
Technical advances, including measurements of baroreflex sensitivity (BRS), heart rate variability (HRV) and the concentration of norepinephrine, allow cardiac autonomic function to be assessed. The reliability coefficients of these parameters, however, were shown to be around $50 \% .{ }^{12} \mathrm{~A}$ reduction in myocardial uptake of ${ }^{123} \mathrm{I}$-metaiodobenzylguanidine (MIBG) reflects a reduction in the concentration of norepinephrine at presynaptic sites or a reduction in the neural density, whereas an enhanced washout rate (WR) of ${ }^{123}$ I-MIBG reflects enhanced release of norepinephrine from presynaptic sites. ${ }^{13}$ Cardiac ${ }^{123} \mathrm{I}-\mathrm{MIBG}$ scintigraphy is a sensitive method for detecting sympathetic dysfunction in many clinical disorders, including diabetes mellitus. ${ }^{14,15}$

We hypothesized that an increased severity of DR is associated with cardiovascular autonomic dysfunction and insulin resistance in type 2 diabetic patients. To test our hypothesis, we compared BRS, HRV, plasma norepinephrine concentrations and cardiac ${ }^{123}$ I-MIBG scintigraphy in addition to the metabolic profiles in Japanese type 2 diabetic patients with and without DR, and independent predictors of the DR in these populations were evaluated.

${ }^{1}$ Department of Cardiology, Oita Red Cross Hospital, Oita, Japan; ${ }^{2}$ Department of Internal Medicine 1, Oita University, Oita, Japan; ${ }^{3}$ Department of Cardiovascular Science, Oita University, Oita, Japan and ${ }^{4}$ Faculty of Medicine, Department of Biostatistics, Oita University, Oita, Japan

Correspondence: Dr F Anan, Department of Cardiology, Oita Red Cross Hospital, 3-2-37 Chiyomachi, Oita 870-0033, Japan.

E-mail: anan-f@med.oita-u.ac.jp

Received 13 August 2008; revised 18 December 2008; accepted 16 January 2009; published online 27 February 2009 


\section{METHODS}

\section{Study population}

We screened 155 consecutive Japanese patients with type 2 diabetes mellitus who were admitted to our department between June 2004 and May 2006.

Among them, 70 patients ( 37 men and 33 women), with a mean s.d. age of $58 \pm 6$ years, fulfilled the inclusion criteria and were enrolled in this study. The inclusion criteria were as follows:

(1) Organic heart disease was not determined by treadmill exercise electrocardiography (ECG). We excluded subjects who have ST-T abnormal change by treadmill exercise ECG.

(2) An absence of causes of secondary hypertension (that is, primary aldosteronism, renal vascular hypertension, hyperthyroidism and pheochromocytoma).

(3) No past history of chronic diseases, such as renal failure (creatinine $>1.5 \mathrm{mg}$ per $100 \mathrm{ml}$ ), pulmonary disease, liver dysfunction (aminotransferase $>50 \mathrm{IUl}^{-1}$ ), arteriosclerosis obliterans, sleep apnea syndrome and symptomatic cerebrovascular disease, was noted.

(4) The patient was not currently receiving treatment with insulin. (For this reason, insulin therapy patients were not measured by the homeostasis model assessment; HOMA index.)

(5) Female patients who were pregnant or treated with any postmenopausal hormone replacement therapy.

Of the 155 screened patients, 85 were excluded from further evaluation due to extenuating circumstances. Of those excluded, 35 patients were being treated with insulin, 7 patients had angina pectoris, 6 patients had renal failure and 6 patients had symptomatic cerebrovascular disease, 6 patients being treated with $\beta$-blockers.

Five patients had arteriosclerotic obliterans, five patients had sleep apnea syndrome, four patients being treated with antiarrhythmia drugs, four patients had secondary hypertension (two patients had primary adlosteronism, one patient had renal vascular hypertension and one patient had hyperthyroidism), three patients had liver dysfunction (one patient had hepatitis B and two patients had hepatitis $\mathrm{C}$ ), two patients had lung cancer and two patients were treated with postmenopausal hormone replacement therapy. Therefore, only 70 patients were selected for the study.

All subjects gave their written informed consent to participate in the study, and the study protocol was approved by the ethics committee of the Oita University Hospital.

\section{Assessment of DR}

Ophthalmologic records, including ophthalmologic charts, fundus photography and fluorescein retinal angiography, were reviewed to evaluate patient's retina. According to a modification of the Diabetic Retinopathy Study and Early Treatment Diabetic Retinopathy Study grading scale, the severity in the worst affected eye was used, and the patients with retinopathy were grouped into three categories of retinopathy: (1) those with mild-to-moderate nonproliferative DR (NPDR) (only microaneurysms or microaneurysms plus one or more of the following: retinal hemorrhages, soft exudates, hard exudates, intraretinal microvascular abnormalities or venous beading), (2) those at a severe stage of NPDR (at least three of the following: extensive retinal hemorrhages or microaneurysms, soft exudates, intraretinal microvascular abnormalities and venous beading in two or more quadrants) and (3) those with proliferative DR (PDR) (the presence of new vessels, preretinal or vitreous hemorrhages, panretinal photocoagulation scars and a history of vitrectomy). ${ }^{16,17}$

\section{Definition of hypertension}

Hypertension was defined by performing BP measurement, registered as the average of three measurements obtained with a mercury-column sphygmomanometer after $10 \mathrm{~min}$ of physical resting by the patients. Essential hypertension was defined as diastolic blood pressure $\geqslant 90 \mathrm{~mm} \mathrm{Hg}$, systolic blood pressure $\geqslant 140 \mathrm{~mm} \mathrm{Hg}$ or self-reported use of antihypertensive medication. ${ }^{18}$

\section{Laboratory methods}

Blood was taken at 0700 hours from the antecubital vein with the patient in the recumbent position after an overnight fast. All patients underwent routine laboratory tests including assays for serum electrolytes, serum total cholesterol, serum triglycerides, serum high-density lipoprotein cholesterol (HDL-C), fasting plasma glucose and fasting immunoreactive insulin. Insulin resistance was evaluated by the HOMA index: (fasting plasma insulin $\left[\mu \mathrm{U} \mathrm{ml}^{-1}\right] \times$ fasting plasma glucose $\left.\left[\mathrm{mmoll}^{-1}\right]\right) / 22.5 .^{19}$ Dyslipidemia was defined as fasting triglyceride levels $\geqslant 200 \mathrm{mg}$ per $100 \mathrm{ml}$ or a HDL-C concentration $<45 \mathrm{mg}$ per $100 \mathrm{ml}$ for women and $<35 \mathrm{mg}$ per $100 \mathrm{ml}$ for men. ${ }^{20}$

\section{Echocardiography}

M-mode and two-dimensional echocardiography and cardiac Doppler recordings were obtained using a phase-array echo-Doppler system. Echocardiograms were obtained using standard parasternal, short axis and apical views. The left ventricular mass was calculated as $1.04 \times\left([\text { LVIDd+IVSTd }+ \text { PWTd }]^{3}-\mathrm{LVIDd}^{3}\right)-$ $14 \mathrm{~g}$, where LVIDd is the left ventricular internal diameter at the end diastole, IVSTd is the intraventricular septal thickness at the end diastole and PWTd is the posterior wall thickness at the end diastole. The left ventricular mass was divided by the body surface area to calculate the left ventricular mass index. Pulsed Doppler recordings were made from a standard apical four-chamber view. Mitral inflow velocity was recorded with the sample volume at the mitral annulus level, taking the average from at least three cardiac cycles. The peak velocity of early $(E)$ and late ventricular filling $(A)$ was determined, and the ratio $(E / A)$ and deceleration time were recorded.

\section{Cardiovascular autonomic function tests}

Autonomic function was assessed according to methods described in earlier studies. ${ }^{9-11}$ During the tests, which were performed between 0900 and 1100 hours, all subjects were in a supine position in a quiet room with dimmed lights. Autonomic function tests were performed in the morning after an overnight $(\geqslant 12 \mathrm{~h})$ fast. For measurement of the plasma norepinephrine concentration, a blood sample was obtained from a catheter inserted in the right cubital vein $30 \mathrm{~min}$ before sampling. We measured plasma norepinephrine concentration according to the methods described in an earlier study. ${ }^{7-9}$ Arterial blood pressure was recorded noninvasively through a tonometric sensor attached over the left radial artery (Jentow-7700; Nihon Colin, Komaki, Japan). The accuracy of continuous blood pressure monitoring has been demonstrated earlier. ${ }^{21}$ Arterial blood pressure and a standard 12-lead ECG were monitored simultaneously; data were stored in a PCM data recorder (RD200T; TEAC, Tokyo, Japan). Three-lead precordial Holter ECG recordings (model 459; Del Mar Avionics, Irvine, CA, USA) were also obtained throughout the procedure for analysis of HRV.

After an interval of $30 \mathrm{~min}$ to permit stabilization of the cardiovascular baroreflex mechanism, the patient was asked to breathe at a rate of 15 breaths per min using a metronome to stabilize the relationship between respiration and cardiovascular function. BRS was assessed by the phenylephrine method. ${ }^{7-9}$ Briefly, phenylephrine (2-3 $\left.\mu \mathrm{g} \mathrm{kg}^{-1}\right)$ was injected for $15 \mathrm{~s}$ to obtain a 15 - to $40-$ $\mathrm{mm} \mathrm{Hg}$ rise in systolic blood pressure. BRS was calculated as the slope of the linear regression function relating systolic blood pressure changes to changes in the RR interval. Regression lines with more than 20 data points and a correlation coefficient $(r)$ greater than 0.8 were accepted for analysis. The average of the two slopes was taken as the BRS value.

Heart rate variability was analyzed using Holter ECG recordings (Marquette Electronics Inc., Milwaukee, WI, USA), with methods described in an earlier study. ${ }^{22}$ The power spectrum of the RR interval was computed by a fast Fourier transformation and expressed as the area under the power spectrum. We calculated the power of two spectral bands: the normal-frequency (LF) component at $0.04-0.15 \mathrm{~Hz}$ and the high-frequency (HF) component at $0.15-0.40 \mathrm{~Hz}$. On the basis of their skewed distribution, the measured values of HRV were transformed to natural logarithmic values. The ratio of LF to HF (LF/HF) also was computed.

Planar and single-photon emission-computed tomography studies were performed $15 \mathrm{~min}$ (early) and $4 \mathrm{~h}$ (delayed) after the injection of $111 \mathrm{MBq}$ of ${ }^{123}$ I-MIBG using a rotating gamma camera (ZLC 7500; Siemens, Munich, Germany). Data were analyzed with analysis software (SCINTIPAC; Shimadzu, 
Kyoto, Japan). The anterior planar images from early and delayed ${ }^{123}$ I-MIBG studies were analyzed visually. For semiquantitative analysis, regions of interest were identified within the whole heart and a $10 \times 10-\mathrm{mm}$ area over the upper mediastinum on the early and delayed planar images was used to calculate the mean heart-to-mediastinum $(H / M)$ ratio. After correcting for the physical decay of ${ }^{123} \mathrm{I}$, the percent $\mathrm{WR}$ of the tracer from the myocardium was determined over a 4 -h period.

\section{Anthropometric and body composition measurement}

The anthropometric and body composition characteristics of the patients were evaluated using the following parameters: height, body weight, body mass index (BMI), waist circumference, hip circumference and waist-to-hip ratio. BMI was calculated as weight/(height $\left.{ }^{2}\right)\left(\mathrm{kg} / \mathrm{m}^{2}\right)$. The waist circumference was measured midway between the lower rib margin and the iliac crest, and the hip circumference was measured at the widest circumference over the trochanter in standing subjects after normal expiration.

\section{Statistical analysis}

All data are classified into two groups, that is the normal and the DR, and are summarized as the means \pm s.d. (Table 1). For each variable in Table 1, the twosided test with a level of significance 0.05 was performed to test the difference between the two groups. The Student's $t$-test was used for continuous variables, and for categorical variables, the $\chi^{2}$ test was carried out. Logistic regression analysis was used to assess the influence of explanatory variables on DR, where the explanatory variables were age, gender, BMI, duration of diabetes, hypertension, dyslipidemia, blood pressure, heart rate, T-cho, triglyceride, HDL-C, fasting plasma glucose (FPG), fasting immunoreactive isulin (F-IRI), HOMA index, hemoglobin Alc, uric acid, creatinine, ejection fraction, left ventricular mass index, $E / A$ ratio, deceleration time, plasma norepinephrine, HF power, LF/HF, BRS, the percent WR of ${ }^{123} \mathrm{I}-\mathrm{MIBG}$ and the $H / M$ ratio at the early and delayed phases after ${ }^{123}$ I-MIBG administration, where gender, hypertension and dyslipidemia were dichotomized as 1 (presence) and 0 (absence) by cutoff values defined in the earlier section.

In the procedure of DR, positive was represented as 1 and negative as 0 . To determine factors among all explanatory variables used, a backward elimination procedure was employed. In the procedure, the BMI, triglyceride, HDL-C, FPG, F-IRI, HOMA index, uric acid, BRS, the percent WR of ${ }^{123} \mathrm{I}-\mathrm{MIBG}$ and the $H / M$ ratio at the early and delayed phases after ${ }^{123}$ I-MIBG administration were determined as significant factors influencing DR.

All the analyses were performed using a standard statistical package (JMP 6.0; SAS Institute, Cary, NC, USA).

\section{Table 1 Fundus findings of type 2 diabetic patients}

\begin{tabular}{lc}
\hline & No. of patients \\
Fundus findings & (\%) \\
\hline No retinopathy & $41(58.6)$ \\
Mild-to-moderate NPDR & $14(20.0)$ \\
Microaneurysms only & 6 \\
Microaneurysms, retinal hemorrhages and hard exudates & 5 \\
Microaneurysms, retinal hemorrhages and soft exudates & 4 \\
Severe stage of NPDR & $9(12.9)$ \\
Extensive microaneurysms, retinal hemorrhages and soft exudates & 5 \\
Extensive microaneurysms, retinal hemorrhages and soft exudates & 4 \\
and intraretinal microvascular abnormalities & \\
PDR & $6(8.6)$ \\
Neovascularization & 2 \\
Preretinal hemorrhage & 1 \\
Vitreous hemorrhage & 1 \\
Panretinal photocoagulation scar & 1 \\
History of vitrectomy & 1 \\
Total & 70 \\
\hline
\end{tabular}

Abbreviations: NPDR, nonproliferative diabetic retinopathy; PDR, proliferative retinopathy.

\section{RESULTS}

Fundus findings of the 70 diabetes patients are shown in Table 1. Forty one $(58.6 \%)$ diabetic patients did not have retinopathy and 29 (41.4\%) had DR. Among the 29 diabetic patients with retinopathy, 14 patients were grouped as having mild-to-moderate NPDR, 9 as having severe NPDR and 6 patients as having PDR.

As shown in Table 2, the mean ages of the DR and no DR (NDR) groups were similar, and there were no significant differences between the groups with respect to gender, duration of diabetes or administered medications. The BMI values, waist circumferences and waist-tohip ratios were larger in the DR group than in the NDR group ( $P=0.0085, P=0.0011$ and $P=0.0113$, respectively). Regarding glucose metabolism, fasting insulin concentrations and HOMA index were higher in the DR group than in the NDR group $(P<0.0001$ and $P<0.0001$, respectively).

Uric acid was higher in the DR group than in the NDR group $(P=0.0257)$. However, there was no significant difference in hemoglobin A1c. With regard to lipid metabolism, serum triglyceride was higher and serum HDL-C was lower in the DR group than in the NDR group ( $P=0.0321$ and $P=0.0189$, respectively), whereas serum total cholesterol showed no significant difference between the two groups. Regarding the renal function, there was no significant difference in the serum creatinine concentration. The hemodynamic data listed in Table 2 were obtained immediately before BRS assessment. The resting

Table 2 Clinical characteristics of studied patients

\begin{tabular}{|c|c|c|c|}
\hline & $N D R$ & $D R$ & P-value \\
\hline Age (years) & $58 \pm 7$ & $58 \pm 5$ & NS \\
\hline Gender (men/women) & $22 / 19$ & $15 / 14$ & NS \\
\hline Duration of diabetes (year) & $6.3 \pm 3.6$ & $6.8 \pm 3.3$ & NS \\
\hline Hypertension (\%) & 63 & 66 & NS \\
\hline Dyslipidemia (\%) & 41 & 45 & NS \\
\hline \multicolumn{4}{|l|}{ Drug use (\%) } \\
\hline Sulfonylurea & 41 & 45 & NS \\
\hline$\alpha$-Glucosidase inhibitors & 37 & 34 & NS \\
\hline Statin & 32 & 34 & NS \\
\hline Aldose reductase inhibitor epalrestat & 25 & 28 & NS \\
\hline Calcium channel antagonists & 39 & 41 & NS \\
\hline ACE inhibitors & 22 & 21 & NS \\
\hline Angiotensin receptor blocker & 44 & 45 & NS \\
\hline Body mass index $\left(\mathrm{kg} / \mathrm{m}^{2}\right)$ & $24.9 \pm 2.3$ & $26.5 \pm 2.6$ & 0.0085 \\
\hline Waist circumferences $(\mathrm{cm})$ & $84.2 \pm 7.3$ & $90.9 \pm 8.5$ & 0.0011 \\
\hline Hip circumferences $(\mathrm{cm})$ & $96.2 \pm 5.5$ & $97.8 \pm 7.9$ & NS \\
\hline Waist-to-hip ratio & $0.88 \pm 0.10$ & $0.93 \pm 0.82$ & 0.0113 \\
\hline Systolic blood pressure $(\mathrm{mm} \mathrm{Hg})$ & $129 \pm 17$ & $132 \pm 12$ & NS \\
\hline Diastolic blood pressure $(\mathrm{mm} \mathrm{Hg})$ & $76 \pm 8$ & $77 \pm 7$ & NS \\
\hline Heart rate (b.p.m.) & $67 \pm 6$ & $68 \pm 8$ & NS \\
\hline Total cholesterol (mg per $100 \mathrm{ml}$ ) & $201 \pm 27$ & $210 \pm 33$ & NS \\
\hline Triglyceride (mg per $100 \mathrm{ml}$ ) & $125 \pm 41$ & $149 \pm 50$ & 0.0321 \\
\hline HDL cholesterol (mg per $100 \mathrm{ml}$ ) & $48 \pm 11$ & $42 \pm 9$ & 0.0189 \\
\hline Fasting plasma glucose (mg per $100 \mathrm{ml}$ ) & $142 \pm 18$ & $153 \pm 22$ & 0.0187 \\
\hline Fasting immunoreactive insulin $\left(\mu \mathrm{Uml}^{-1}\right)$ & $5.8 \pm 1.1$ & $8.1 \pm 2.7$ & $<0.0001$ \\
\hline HOMA index & $2.0 \pm 0.4$ & $3.1 \pm 1.1$ & $<0.0001$ \\
\hline Hemoglobin A1c (\%) & $7.5 \pm 1.3$ & $7.8 \pm 1.1$ & NS \\
\hline Uric acid (mg per $100 \mathrm{ml}$ ) & $5.7 \pm 1.2$ & $6.4 \pm 1.0$ & 0.0257 \\
\hline Creatinine (mg per $100 \mathrm{ml}$ ) & $0.7 \pm 0.2$ & $0.8 \pm 0.2$ & NS \\
\hline
\end{tabular}

Abbreviations: ACE, angiotensin-converting enzyme; DR, diabetic retinopathy; $H D L$, high-density lipoprotein; HOMA, homeostasis model assessment; NDR, no diabetic retinopathy; NS, not significant.

Data are mean \pm s.d. 
heart rate, systolic and diastolic blood pressures were not significantly different between the two groups.

Table 3 presents a summary of echocardiographic findings. The left ventricular dimensions at end diastole and end systole, intraventricular septal and posterior wall thickness at end diastole, ejection fraction and left ventricular mass index were essentially similar in the two groups. With regard to the left ventricular diastolic function, the ratio of peak velocities of early to late ventricular filling ( $E / A$ ratio) was lower in the DR group than in the NDR group $(P=0.0476)$. Deceleration time was longer in the DR group than in the NDR group $(P=0.0485)$.

Figure 1 summarizes the results of the cardiovascular autonomic function tests. BRS was lower in the DR group than in the NDR group

Table 3 Echocardiographic findings

\begin{tabular}{|c|c|c|c|}
\hline & $N D R$ & $D R$ & P-value \\
\hline Ejection fraction (\%) & $70 \pm 6$ & $68 \pm 5$ & NS \\
\hline LVIDd (mm) & $47 \pm 3$ & $49 \pm 4$ & NS \\
\hline LVIDs (mm) & $30 \pm 3$ & $32 \pm 4$ & NS \\
\hline IVSTd (mm) & $8.7 \pm 1.1$ & $9.0 \pm 1.3$ & NS \\
\hline PWTd (mm) & $9.2 \pm 1.2$ & $9.5 \pm 1.0$ & NS \\
\hline LVMI $\left(\mathrm{g} \mathrm{m}^{-2}\right)$ & $109 \pm 19$ & $116 \pm 22$ & NS \\
\hline$E / A$ ratio & $0.96 \pm 0.18$ & $0.87 \pm 0.15$ & 0.0476 \\
\hline Deceleration time (ms) & $236 \pm 26$ & $249 \pm 31$ & 0.0485 \\
\hline
\end{tabular}

Abbreviations: $D R$, diabetic retinopathy; $E / A$, the ratio of peak velocities of early to late ventricular filling; IVSTd, interventricular septal thickness at end diastole; LVIDd, left ventricular internal dimension at end diastole; LVIDs, left ventricular internal dimension at end systole; LVMI, left ventricular mass index; NDR, no diabetic retinopathy; NS, not significant; PWTd, posterior wall thickness at end diastole.

Data are mean \pm s.d.
(DR group, $8.8 \pm 4.0 \mathrm{~ms}$ per $\mathrm{mm} \mathrm{Hg} v s$. NDR group, $11.4 \pm 3.1 \mathrm{~ms}$ per $\mathrm{mm} \mathrm{Hg} P=0.0028$; Figure 1a). Plasma norepinephrine concentration was similar in both groups (DR group, $229 \pm 80 \mathrm{pg} \mathrm{ml}^{-1}$ vs. NDR group, $212 \pm 90 \mathrm{pg} \mathrm{ml}^{-1}$; $P=0.5146$; Figure $1 \mathrm{~b}$ ). Furthermore, analysis of HRV in the DR and NDR groups revealed that the HF power (3.5 \pm 1.4 and $4.0 \pm 1.6 \mathrm{ln}-\mathrm{ms}^{2}$, respectively; $\left.P=0.1803\right)$ and the $\mathrm{LF} / \mathrm{HF}$ ratios ( $1.3 \pm 1.0$ and $1.5 \pm 1.1$, respectively; $P=0.3423$; Figure 1c) were not significantly different between the two groups. Cardiac ${ }^{123}$ I-MIBG scintigraphy disclosed that the $H / M$ ratios at early and delayed phases in the DR group were significantly smaller than those in the NDR group (early phase: $2.05 \pm 0.27$ vs. $2.26 \pm 0.22$, respectively; $P=0.0004$; delayed phase: $1.91 \pm 0.29$ vs. $2.18 \pm 0.21$, respectively; $P<0.0001$; Figure 1d).

The percent WR of ${ }^{123} \mathrm{I}-\mathrm{MIBG}$ was higher in the DR group than in the NDR group (45.1 \pm 8.9 vs. $34.5 \pm 9.6 \%, P<0.0001$; Figure $1 d$ ).

In univariate logistic regression analysis, the DR was associated with triglyceride (odds ratio (OR) 1.01, 95\% CI=1.00-1.03; $P=0.0382$ ), HDL-C (OR 0.94, 95\% CI $=0.90-0.99 ; P=0.0207$ ), fasting plasma glucose ( $\mathrm{OR} 1.03,95 \% \mathrm{CI}=1.00-1.06 ; P=0.0227$ ), fasting plasma insulin (OR 1.87, 95\% $\mathrm{CI}=1.32-2.65 ; P=0.0004$ ), HOMA index (OR 6.05, 95\% CI=2.28-16.1; $P=0.0003$ ), uric acid (OR 1.65, 95\% $\mathrm{CI}=1.05-2.60 ; \quad P=0.0310)$, BRS $(\mathrm{OR} \quad 0.81, \quad 95 \% \quad \mathrm{CI}=0.69-0.94$; $P=0.0055), H / M$ ratio at early phase (OR $0.63,95 \% \mathrm{CI}=0.42-0.86$; $P=0.0039), H / M$ ratio at delayed phase (OR $0.55,95 \% \mathrm{CI}=0.31-0.83$; $P=0.0008$ ) and the percent WR of ${ }^{123} \mathrm{I}$-MIBG (OR 1.13, 95\% $\mathrm{CI}=1.06-1.20 ; P=0.0002)$ as the dependent lipid and glucose metabolic and cardiovascular autonomic function parameters in type 2 diabetic patients (Table 4).

Multivariate logistic analysis identified HOMA index (OR 4.36, 95\% $\mathrm{CI}=1.53-12.5 ; P=0.0060)$ and the percent WR of ${ }^{123} \mathrm{I}-\mathrm{MIBG}$
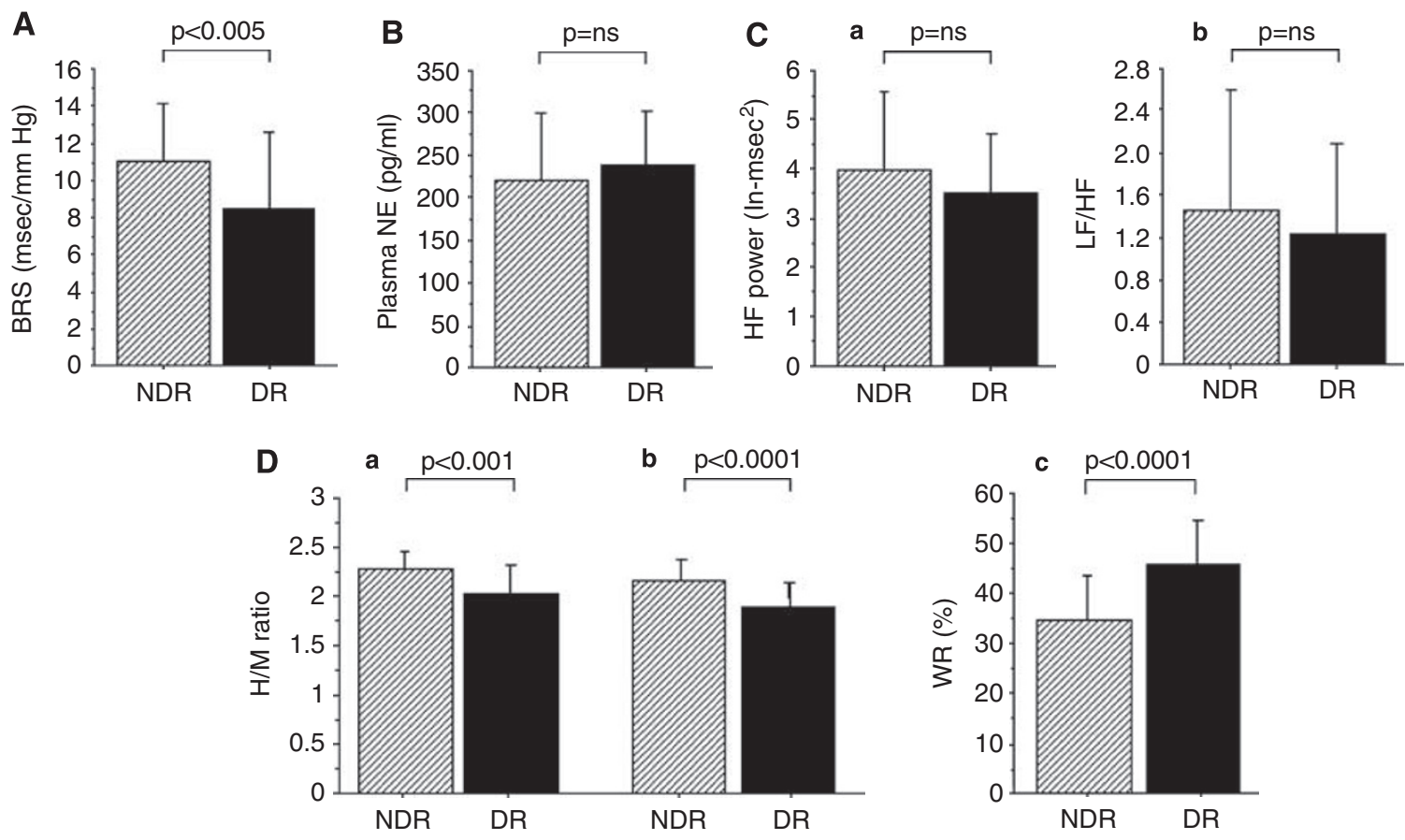

Figure 1 Comparison of autonomic function tests between type 2 diabetic patients with diabetic retinopathy (DR) and those with no diabetic retinopathy (NDR). (A) Baroreflex sensitivity (BRS). (B) Plasma norepinephrine (NE) concentration. (C) Heart rate variability (HRV). Power of high-frequency component $(\mathrm{HF}, 0.15-0.40 \mathrm{~Hz}, \mathrm{a})$ and the ratio of the low-frequency power $(\mathrm{LF}, 0.04-0.15 \mathrm{~Hz})$ to $\mathrm{HF}$ power $(\mathrm{LF} / \mathrm{HF}, \mathrm{b})$. The distribution of $\mathrm{HRV}$ values was skewed and the values were thus transformed to natural logarithmic values. (D) Cardiac ${ }^{123}$-metaiodobenzylguanidine (MIBG) scintigraphic findings. Myocardial uptake of ${ }^{123}$ I-MIBG at early (a) and delayed (b) phases. Myocardial uptake of ${ }^{123}$ I-MIBG is expressed as the mean heart-to-mediastinum (H/M) ratio. (c) Percent washout rate (WR) of ${ }^{123}$ I-MIBG. Data are mean \pm s.d. NS=not significant. 
Table 4 Univariate logistic regression analysis with diabetic retinopathy as the dependent variable in type 2 diabetes mellitus

\begin{tabular}{|c|c|c|c|}
\hline & \multicolumn{3}{|c|}{ Diabetic retinopathy } \\
\hline & Odds ratio & $95 \% \mathrm{Cl}$ & $\mathrm{P}$-value \\
\hline Age & 0.98 & $0.90-1.07$ & NS \\
\hline Gender & 2.15 & $0.63-7.36$ & NS \\
\hline BMI & 1.33 & $1.06-1.66$ & 0.0128 \\
\hline Duration of diabetes & 1.04 & $0.91-1.20$ & NS \\
\hline Hypertension & 0.85 & $0.36-2.59$ & NS \\
\hline Dyslipidemia & 1.13 & $0.53-2.65$ & NS \\
\hline Systolic blood pressure & 1.01 & $0.98-1.05$ & NS \\
\hline Diastolic blood pressure & 1.03 & $0.97-1.10$ & NS \\
\hline Heart rate & 1.02 & $0.95-1.09$ & NS \\
\hline T-cholesterol & 1.01 & $0.99-1.03$ & NS \\
\hline Triglyceride & 1.01 & $1.00-1.03$ & 0.0382 \\
\hline HDL cholesterol & 0.94 & $0.90-0.99$ & 0.0207 \\
\hline Fasting plasma glucose & 1.03 & $1.00-1.06$ & 0.0227 \\
\hline F-IRI & 1.87 & $1.32-2.65$ & 0.0004 \\
\hline HOMA index & 6.05 & $2.28-16.1$ & 0.0003 \\
\hline Hemoglobin A1c & 1.26 & $0.83-1.93$ & NS \\
\hline Uric acid & 1.65 & $1.05-2.60$ & 0.0310 \\
\hline Creatinine & 1.68 & $0.13-21.6$ & NS \\
\hline Ejection fraction & 0.96 & $0.86-1.08$ & NS \\
\hline LVMI & 1.02 & $0.99-1.05$ & NS \\
\hline$E / A$ ratio & 0.79 & $0.61-1.08$ & NS \\
\hline Deceleration time & 1.03 & $0.99-1.05$ & NS \\
\hline Plasma norepinephrine & 1.00 & $0.98-1.01$ & NS \\
\hline HF power & 0.83 & $0.58-1.11$ & NS \\
\hline $\mathrm{LF} / \mathrm{HF}$ & 0.78 & $0.51-1.26$ & NS \\
\hline Baroreflex sensitivity & 0.81 & $0.69-0.94$ & 0.0055 \\
\hline$H / M$ ratio at early phase & 0.63 & $0.42-0.86$ & 0.0039 \\
\hline$H / M$ ratio at delay phase & 0.55 & $0.31-0.83$ & 0.0008 \\
\hline Wash out & 1.13 & $1.06-1.20$ & 0.0002 \\
\hline
\end{tabular}

Abbreviations: $\mathrm{BMI}$, body mass index; $\mathrm{Cl}$, confidence interval; $E / A$, the ratio of peak velocities of early to late ventricular filling: $H / M$ ratio, heart-to-mediastinum ratio; $H D L$, high-density lipoprotein; HF, high-frequency; HOMA, homeostasis model assessment; LF/HF, ratio of LF to HF; LVMI, left ventricular mass index; NDR, no diabetic retinopathy; NS, not significant. Significant predictors of diabetic retinopathy were explored among three parameters: gender (female $=0$, men $=1$ ), hypertension (absent $=0$, present $=1$ ) and dyslipidemia (absent $=0$, present $=1$ ).

(OR $1.45,95 \% \mathrm{CI}=1.08-2.37 ; P=0.0270)$ in type 2 diabetic patients as independent parameters for DR (Table 5).

\section{DISCUSSION}

In our present study, type 2 diabetic patients with DR group had lower BRS, and lower myocardial uptake and enhanced clearance of ${ }^{123}$ I-MIBG, relative to the values in type 2 diabetic patients with NDR group. Among the metabolic profiles, the fasting plasma insulin concentration and the HOMA index were higher in patients with DR group than in those with NDR group. Multiple logistic analyses revealed that the HOMA index and the percent WR of ${ }^{123}$ I-MIBG were independent risk factors for the presence of DR in type 2 diabetic patients.

This study revealed the novel and important findings that the type 2 diabetic patients in the DR group had the higher percent WR of ${ }^{123}$ I-MIBG and stronger insulin resistance than the NDR group.

There is a report indicating that DR is associated with insulin resistance in type 1 diabetes $^{10}$ and type 2 diabetes mellitus. ${ }^{11}$

Hadjadj et al. ${ }^{10}$ investigated the association between DR and insulin resistance score using a WHO recommendation ${ }^{23}$ (that is, hyperten-
Table 5 Multivariate logistic regression analysis with WML as dependent variable in type 2 diabetes mellitus

\begin{tabular}{lccr}
\hline & \multicolumn{3}{c}{ White matter lesions } \\
\cline { 2 - 4 } & Odds ratio & $95 \% \mathrm{Cl}$ & P-value \\
\hline HOMA index & 4.36 & $1.53-12.5$ & 0.0060 \\
The percent WR of ${ }^{123}$ I-MIBG & 1.45 & $1.08-2.37$ & 0.0270 \\
\hline $\begin{array}{l}\text { Abbreviations: Cl, confidence interval; HOMA, homeostasis model assessment; MIBG, } \\
\text { metaiodobenzylguanidine; WR, washout rate. }\end{array}$ & &
\end{tabular}

sion, personal history of lipid disorders, personal history of type 2 diabetes and obesity were considered). They found a significant association of DR with insulin resistance in type 1 diabetic patients. Parvanova et al. ${ }^{11}$ have reported that proliferative retinopathy is associated with insulin resistance using a hyperinsulinemic-euglycemic clamp in 115 patients with type 2 diabetes mellitus. In this study, the DR was associated with BMI, triglyceride levels, HDL-C levels, fasting plasma glucose, fasting plasma insulin concentration and the HOMA index values.

The mechanism by which DR increases insulin resistance remains to be elucidated. In our opinion, there are a couple of possible explanations for this observation. First, defective fibrinolysis caused by excess plasminogen activator inhibitor-1 activity and selective inhibition of some antiatherogenic effects of insulin may promote the occlusion of retinal capillaries and secondary ischemia-induced neovascularization. ${ }^{24}$ Second, the ischemic damage can be further amplified by insulin resistance that has been related to a lower ability of insulin to induce vasodilatation through impaired nitric oxide endothelial production or accelerated inactivation. ${ }^{25}$

The relationship between DR and cardiovascular autonomic function has been examined in earlier studies using HRV analysis. ${ }^{26,27}$ Schmidt et al. ${ }^{26}$ have shown that DR is associated with heart rate response by electrocardiographical monitoring in type 2 diabetic patients. Duvnjak et al. ${ }^{27}$ reported that DR is related to sympathetic and parasympathetic reactivity by HRV. In this study, HRV and the plasma norepinephrine concentrations were not different between the two groups. However, a significant difference was seen in ${ }^{123}$ I-MIBG parameters. These results may suggest association of DR in diabetic patients with impairment of uptake-1 (norepinephrine transporter) as well as the acceleration of norepinephrine turnover in sympathetic nerve terminal. Impairment in uptake- 1 in diabetic rats has been reported. ${ }^{28}$ Our and the earlier studies by others ${ }^{7-9,29}$ demonstrated that ${ }^{123}$ I-MIBG scintigraphy is a fairly sensitive method for detecting cardiac sympathetic dysfunction in diabetic patients. The present results support potential of ${ }^{123}$ I-MIBG scintigraphy in the diagnosis of cardiovascular autonomic dysfunction.

Although the precise mechanisms underlying the interactions between DR and impaired autonomic function remain unclear, there are several explanations regarding the mechanisms. First, DR may affect autonomic function through endothelial dysfunction and impairment of the nitrate oxide system. DR is associated with endothelial dysfunction by oxidant stress. ${ }^{30}$ Endothelial dysfunction is associated with cardiac autonomic dysfunction and increased HRV. $^{31,32}$ Moreover, we have recently reported that the HOMA index and the myocardial uptake of ${ }^{123}$ I-MIBG at the delayed phase were independent predictors of adiponectin concentration. ${ }^{7}$ In fact, in a recent report demonstrating the association between $\mathrm{DR}$, insulin resistance and adiponectin, the authors stressed the central role of endothelial dysfunction. ${ }^{33}$ Second, DR may cause dysregulation of autonomic nervous system through an enhanced release of free fatty 
acid. Insulin resistance is strongly associated with DR and free fatty acids. $^{34}$

Taken together, it is possible that DR, insulin resistance and autonomic dysfunction interact and reinforce each other through mechanisms associated with endothelial dysfunction.

Compared with the NDR group, patients in the DR group showed cardiac diastolic dysfunction. Consistent with these results, the earlier study demonstrated that cardiac diastolic dysfunction was associated with cardiovascular autonomic dysfunction and insulin resistance. ${ }^{20}$ Annonu et al. ${ }^{35}$ reported that an increased DR was associated with cardiac left ventricular diastolic dysfunction in diabetic patients. Although the precise mechanism is unclear, diastolic dysfunction may interact with cardiac sympathetic nervous function through insulin resistance.

There are several limitations to this study. First, subjects in this study population had essential hypertension, which was treated with one or more antihypertensive drugs. These characteristics of the patients' backgrounds have been reported to affect insulin resistance ${ }^{36,37}$ and sympathetic nerve function. ${ }^{38,39}$ Nonetheless, the results indicate that $\mathrm{DR}$ in diabetic patients is related to cardiac depression and insulin resistance. Second, there is a potential link of DR with an enhanced release of free fatty acid. ${ }^{40}$ It remains uncertain whether free fatty acid status affected the risk of DR in type 2 diabetes patients, because we did not examine the free fatty acid concentration in this investigation. Third, an aldose reductase inhibitor, epalrestat, has been proven to improve peripheral sensory nerve impairments in diabetes mellitus. ${ }^{41}$ Further clinical investigators are needed to determine the relationship between aldose reductase inhibitor, epalrestat, cardiac autonomic function and DR in type 2 diabetes patients. Fourth, we have reported earlier that visceral fat accumulation and hyperhomocysteine are associated with insulin resistance as well as cardiac sympathetic nerve function assessed by ${ }^{123}$ I-MIBG. ${ }^{8,9}$ Therefore, further studies are required to evaluate the association among the DR, visceral fat accumulation, hyperhomocysteine, HOMA index values and ${ }^{123}$ I-MIBG parameters. In addition, the prognostic implications of cardiac autonomic function as assessed by ${ }^{123}$ I-MIBG scintigraphy remain to be addressed, although there are prognostic studies of heart failure patients using cardiac ${ }^{123}$ I-MIBG imaging. ${ }^{42}$ Finally, compared with the mild-to-moderate to severe NPDR group, patients with PDR group showed impaired metabolic/cardiac parameters. The HOMA index values and ${ }^{123}$ I-MIBG parameters were higher in the PDR group than in the mild-to-moderate to severe NPDR group (data not shown). It would be necessary for further studies to examine the independent factor between PDR and NPDR groups according to the impaired metabolic/cardiac parameters.

In conclusion, our findings suggest that DR in patients with type 2 diabetes is associated with both cardiovascular autonomic function assessed by the percent WR of ${ }^{123}$ I-MIBG and insulin resistance. In the future, large cohort studies including other populations may be beneficial.

\section{CONFLICT OF INTEREST}

The authors declare no conflict of interest.

1 Klein R, Klein BE, Moss SE, Cruickshanks KJ. Association of ocular disease and mortality in a diabetic population. Arch Ophthalmol 1999; 117: 1487-1495.

2 Rajala U, Pajunpää H, Koskela P, Keinänen-Kiukaanniemi S. High cardiovascular disease mortality in subjects with visual impairment caused by diabetic retinopathy. Diabetes Care 2000; 23: 957-961.
3 van Hecke MV, Dekker JM, Stehouwer CD, Polak BC, Fuller JH, Sjolie AK, Kofinis A, Rottiers R, Porta M, Chaturvedi N, EURODIAB prospective complications study. Diabetic retinopathy is associated with mortality and cardiovascular disease incidence: the EURODIAB prospective complications study. Diabetes Care 2005; 28: 1383-1389.

4 Juutilainen A, Lehto $S$, Rönnemaa T, Pyörälä K, Laakso M. Retinopathy predicts cardiovascular mortality in type 2 diabetic men and women. Diabetes Care 2007; 30: 292-299.

5 Tsuji H, Larson MG, Venditti Jr FJ, Manders ES, Evans JC, Feldman CL, Levy D. The Framingham Study. Circulation 1996; 94: 2850-2855.

6 La Rovere MT, Bigger Jr JT, Marcus FI, Mortara A, Schwartz PJ. Baroreflex sensitivity and heart-rate variability in prediction of total cardiac mortality after myocardial infraction. ATRAMI (Autonomic Tone and Reflexes After Myocardial Infraction) Investigators. Lancet 1998; 351: 478-484.

7 Takahashi N, Anan F, Nakagawa M, Yufu K, Shinohara T, Tsubone T, Goto K, Masaki T, Katsuragi I, Tanaka K, Kakuma T, Hara M, Saikawa T, Yoshimatsu H. Hypoadiponectinemia in type 2 diabetes mellitus in men is associated with sympathetic overactivity as evaluated by cardiac ${ }^{123}$ I-metaiodobenzylguanidine scintigraphy. Metabolism 2007; 56: 919-924.

8 Anan F, Yonemochi H, Masaki T, Takahashi N, Nakagawa M, Eshima N, Saikawa T, Yoshimatsu $\mathrm{H}$. Homocysteine levels are associated with the results of ${ }^{123} \mathrm{I}$-metaiodobenzylguanidine myocardial scintigraphy in type 2 diabetic patients. Eur J Nucl Med Mol Imaging 2007; 34: 28-35.

9 Anan F, Masaki T, Yonemochi H, Takahashi N, Nakagawa M, Eshima N, Saikawa T, Yoshimatsu $\mathrm{H}$. Abdominal visceral fat accumulation is associated with the results of 123/-metaiodobenzylguanidine myocardial scintigraphy in type 2 diabetic patients. Eur J Nucl Med Mol Imaging 2007; 34: 1189-1197.

10 Hadjadj S, Péan F, Gallois Y, Passa P, Aubert R, Weekers L, Rigalleau V, Bauduceau B, Bekherraz A, Roussel R, Dussol B, Rodier M, Marechaud R, Lefebvre PJ, Marre M. Genesis France-Belgium Study. Different patterns of insulin resistance in relatives of type 2 diabetic patients with retinopathy or nephropathy. Diabetes Care 2004; 27: 2661-2668

11 Parvanova A, Iliev I, Filipponi M, Dimitrov BD, Vedovato M, Tiengo A, Trevisan R, Remuzzi G, Ruggenenti P. Insulin resistance and proliferative retinopathy crosssectional, case-control study in 115 patients with type 2 diabetes. J Clin Endorinol Metab 2004; 89: 4371-4376.

12 Gerritsen J, TenVoorde BJ, Dekker JM, Kingma R, Kostense PJ, Bouter LM, Heethaar RM. Measures of cardiovascular autonomic dysfunction nervous function: agreement, reproducibility, and reference values in middle age and elderly subjects. Diabetologia 2003: 46: 330-338.

13 Sakata K, Shirotani M, Yoshida H, Kurata C. Cardiac sympathetic nervous system in early essential hypertension assessed by ${ }^{123}$ I-MIBG. J Nucl Med 1999; 40: 6-11.

14 Spallone V, Menzinger G. Diagnosis of cardiovascular autonomic neuropathy in diabetes. Diabetes 1997; 46: S67-S76.

15 Watanabe K, Sekiya M, Tsuruoka T, Funada J, Kameoka H, Miyagawa M, Kohara K. Relationship between insulin resistance and cardiac sympathetic nervous function in essential hypertension. J Hypertens 1999; 17: 1161-1168.

16 Diabetic Retinopathy Study Research Group. Report 7: a modification of the Airlie House classification of diabetic retinopathy. Invest Ophthalmol Vis Sci 1981; 21: 210-226.

17 Early Treatment Diabetic Retinopathy Study Research Group. Fundus photographic risk factors for progression of diabetic retinopathy: ETDRS report number 12. Ophthalmology 1991; 98: 823-833.

18 Mancia G, De Backer G, Dominiczak A, Cifkova R, Fagard R, Germano G, Grassi G, Heagerty AM, Kjeldsen SE, Laurent S, Narkiewicz K, Ruilope L, Rynkiewicz A, Schmieder RE, Boudier HA, Zanchetti A. ESH-ESC Task Force on the Management of Arterial Hypertension 2007 ESH-ESC practice guidelines for the management of arterial hypertension: ESH-ESC task force on the management of arterial hypertension. J Hypertens 2007; 25: 1751-1762.

19 Matthews DR, Hosker JP, Rudenski AS, Naylor BA, Treacher DF, Turner RC. Homeostasis model assessment: insulin resistance and beta-cell function from fasting plasma glucose and insulin concentrations in man. Diabetologia 1985; 28: 412-419.

20 Liao D, Sloan RP, Cascio WE, Folsom AR, Liese AD, Evans GW, Cai J, Sharrett AR. Multiple metabolic syndrome is associated with lower heart rate variability. The Atherosclerosis Risk in Communities Study. Diabetes Care 1998; 21: 2116-2122.

21 Sato T, Nishinaga M, Kawamoto A, Ozawa T, Takatsuji H. Accuracy of a continuous blood pressure monitor based on arterial tonometry. Hypertension 1993; 21: 866-874.

22 Malliani A, Pagani M, Lombardi F, Cerutti S. Cardiovascular neural regulation explored in the frequency domain. Circulation 1991; 84: 482-492.

23 Alberti KG, Zimmet PZ. Definition, diagnosis and classification of diabetes mellitus and its complications. Part 1: diagnosis and classification of diabetes mellitus provisional report of a WHO consultation. Diabet Med 1998; 15: 539-553.

24 Yudkin JS. Abnormalities of coagulation and fibrinolysis in insulin resistance. Diabetes Care 1999; 22: C25-C30.

25 Kurioka S, Koshimura K, Murakami Y, Nishiki M, Kato Y. Reverse correlation between urine nitric oxide metabolites and insulin resistance in patients with type 2 diabetes mellitus. Endocr J 2000; 47: 77-81.

26 Schmid H, Schaan B, Cecconello F, Maestri T, Neumann C. Proliferative diabetic retinopathy is related to cardiovascular autonomic neuropathy in non-insulin-dependent diabetes mellitus. Diabtes Res Clin Pract 1995; 29: 163-168.

27 Duvnjak L, Vuckoviæ S, Pepeonik Z, Metelko Z. Relationship between autonomic neuropathy, 24-hr blood pressure and retinopathy in normoalbuminuric and normotensive Type 1 diabetic patients. Diabetes Nutr Metab 2003; 16: 102-108. 
28 Kinjyo Y, Kajiyama S, Fujiwara H, Kanegawa N, Saji H. Influence of the polyol pathway on transporter reduction in diabetic cardiac sympathetic nerve: implication for heterogeneous accumulation of MIBG. Eur J Nucl Med Imaging 2005; 32: 438-442.

29 Mäntysaari M, Kuikka J, Mustonen J, Tahvanainen K, Vanninen E, Länsimies E, Uusitupa $M$. Noninvasive detection of cardiac sympathetic nervous dysfunction in diabetic patients using [123|] metaiodobenzylguanidine. Diabetes 1992; 41: 1069-1075.

30 Abiko T, Abiko A, Clermont AC, Shoelson B, Horio N, Takahashi J, Adamis AP, King GL, Bursell SE. Characterization of retinal leukostasis and hemodynamics in insulin resistance and diabetes: role of oxidants and protein kinase-C activation. Diabetes 2003; 52: 829-837.

31 Ghiadoni L, Donald AE, Cropley M, Mullen MJ, Oakley G, Taylor M, O'Connor G, Betteridge J, Klein N, Steptoe A, Deanfield JE. Mental stress induces transient endothelial dysfunction in humans. Circulation 2000; 102: 2473-2478.

32 Hijmering ML, Stroes ES, Olijhoek J, Hutten BA, Blankestijn PJ, Rabelink TJ. Sympathetic activation markedly reduces endothelium-dependent, flow-mediated vasodilatation. J Am Coll Cardiol 2002; 39: 683-688.

33 Yilmaz MI, Sonmez A, Acikel C, Celik T, Bingol N, Pinar M, Bayraktar Z, Ozata M. Adiponectin may play a part in the pathogenesis of diabetic retinopathy. Eur $J$ Endocrinol 2004; 151: 135-140.

34 Singleton JR, Smith AG, Russell JW, Feldman EL. Microvascular complications of impaired glucose tolerance. Diabetes 2003; 52: 2867-2873.

35 Annonu AK, Fattah AA, Mokhtar MS, Ghareeb S, Elhendy A. Left ventricular systolic and diastolic in asymptomatic patients with non-insulin-dependent diabetes mellitus. J Am Soc Echocardiogr 2001; 14: 885-891.
36 Gavras HP. Issues in hypertension: drug tolerability and special populations. Am J Hypertens 2001; 14 (Part 2): 231S-236S

37 Lender D, Arauz-Pacheco C, Breen L, Mora-Mora P, Ramirez LC, Raskin P. A double blind comparison of the effects of amlodipine and enalapril on insulin sensitivity in hypertensive patients. Am J Hypertens 1999; 12: 298-303.

38 Kontopoulos AG, Athyros VG, Didangelos TP, Papageorgiou AA, Avramidis MJ, Mayroudi MC, Karamitsos DT. Effect of chronic quinapril administration on heart rate variability in patients with diabetic autonomic neuropathy. Diabetes Care 1997; 20: 355-361.

39 Rodgers JE, Patterson JH. Angiotensin II-receptor blockers: clinical relevance and therapeutic role. Am J Health Syst Pharm 2001; 58: 671-683.

40 Huang EJ, Kuo WW, Chen YJ, Chen TH, Chang MH, Lu MC, Tzang BS, Hsu HH, Huang CY, Lee SD. Homocysteine and other biochemical parameters in type 2 diabetes mellitus with different diabetic duration or diabetic retinopathy. Clin Chim Acta 2006; 366: 293-298.

41 Hotta N, Akanuma Y, Kawamori R, Matsuoka K, Oka Y, Shichiri M, Toyota T, Nakashima M, Yoshimura I, Sakamoto N, Shigeta Y. Long-term clinical effects on epalrestat, an aldose reductase inhibitor, on diabetic peripheral neuropathy: the 3-year, multicenter, comparative Aldose Reductase Inhibitor-Diabetes Complications Trial. Diabetes Care 2006; 29: 1538-1544.

42 Yamashina S, Yamazaki J. Role of MIBG myocardial scintigraphy in the assessment of heart failure: the need to establish evidence. Eur J Nucl Med Mol Imaging 2004; 31: $1353-1355$ 